\title{
AVALIAÇÃO PROSPECTIVA DA EVOLUÇÃO FUNCIONAL DE PACIENTES TRATADOS DE ESTENOSE DE CANAL LOMBAR COM DESCOMPRESSÃO SEM ARTRODESE
}

\author{
PROSPECTIVE EVALUATION OF FUNCTIONAL EVOLUTION OF PATIENTS TREATED OF \\ LUMBAR CANAL STENOSIS WITH DECOMPRESSION WITHOUT ARTHRODESIS
}

\section{EVALUACIÓN PROSPECTIVA DE LA EVOLUCIÓN FUNCIONAL DE LOS PACIENTES TRATADOS DE ESTENOSIS DEL CANAL LUMBAR CON DESCOMPRESIÓN SIN ARTRODESIS}

Frederico Araújo leite ${ }^{1}$, Diogo Barbosa de Carvalho ${ }^{1}$, Carlos Eduardo Gonçales Barsottil ${ }^{2}$. Francisco Prado Eugênio dos Santos², Carlos Eduardo Algavez Soares de Oliveira ${ }^{3}$

\begin{abstract}
RESUMO
Objetivo: Avaliar se o procedimento cirúrgico com descompressão posterior sem artrodese da coluna lombar traz benefícios em avaliações prospectivas de dor e incapacidade. Métodos: Dezesseis pacientes foram avaliados através de questionários de incapacidade e dor no período pré-operatório e durante o seguimento pós-operatório de um mês, seis meses e um ano, tendo sido realizada cirurgia de descompressão sem instrumentação pedicular ou artrodese. Resultados: Foram observadas melhoras nos medidores de dor e incapacidade durante o seguimento de um ano pós-operatório em relação aos valores iniciais. Conclusão: A descompressão sem artrodese em pacientes com estenose de canal lombar demonstrou ser eficaz na melhora da dor e incapacidade em pacientes selecionados no seguimento de um ano pós-operatório.
\end{abstract}

Descritores: Medição da dor; Coluna vertebral; Estenose espinal/cirurgia.

\begin{abstract}
Objective: To evaluate whether the surgical procedure with posterior decompression without fusion of the lumbar spine is beneficial in prospective evaluations of pain and disability. Methods: Sixteen patients were evaluated through questionnaires of disability and pain preoperatively and during the postoperative follow-up at one month, six months, and one year, carrying out surgical decompression surgery without pedicle instrumentation or arthrodesis. Results: There were improvements in measurements of pain and disability during follow-up one year after surgery compared to baseline. Conclusion: The decompression without fusion in patients with lumbar canal stenosis proved to be effective in improving pain and disability in selected patients one year postoperatively.
\end{abstract}

Keywords: Pain measurement; Spine; Spinal stenosis/surgery.

\section{RESUMEN}

Objetivo: Evaluar si el procedimiento quirúrgico con descompresión posterior sin fusión de la columna lumbar es beneficioso en análisis prospectivos de dolor y discapacidad. Métodos: Dieciséis pacientes fueron evaluados por cuestionarios de discapacidad y dolor en el preoperatorio y durante el seguimiento postoperatorio de un mes, seis meses y un año, después de llevar a cabo la cirugía de descompresión sin instrumentación pedicular o artrodesis. Resultados: Se observó una mejoría en el dolor y la discapacidad según la medición durante el seguimiento un año después de la cirugía en comparación con el valor basal. Conclusión: La descompresión sin fusión en pacientes con estenosis del canal lumbar demostró ser eficaz para mejorar el dolor y la discapacidad en pacientes seleccionados en el seguimiento un año después de la operación.

Descriptores: Dimensión del dolor; Columna vertebral; Estenosis espinal/cirugía.

\section{INTRODUÇÃO}

A estenose do canal lombar (ECL) é uma condição dolorosa e potencialmente incapacitante comumente encontrada acometendo principalmente a população idosa, que atualmente apresenta um potencial significativo de crescimento ${ }^{1}$. É uma causa frequente de lombalgia, sendo a principal indicação para cirurgia de coluna lombar em pacientes com mais de 65 anos nos Estados Unidos ${ }^{2,3}$.

A estenose de canal vertebral tem diversas causas, divididas em congênitas, adquiridas e combinadas ${ }^{4}$. A síndrome clínica da ECL é mais frequentemente secundária as alterações degenerativas na população idosa ${ }^{5}$. Estas alterações incluem a redução na espessura das cartilagens articulares, formação osteofitária, esclerose subcondral, hipertrofia facetária e do ligamento amarelo, resultando na redução volumétrica do canal vertebral. O processo degenerativo é lento e progressivo. Os sintomas mais frequentes são lombalgia, claudicação, radiculopatia e as disfunções urinárias. A claudicação neurogênica intermitente é a principal causa de comprometimento de mobilidade e perda de independência em idosos ${ }^{6}$

O diagnóstico é realizado através da história clínica, exame físico, radiografias da coluna nas incidências anteroposterior e perfil, avaliações radiográficas dinâmicas, estudos eletrofisiológicos e ressonância magnética. A ressonância magnética é o exame considerado de excelência para a avaliação precisa dos níveis envolvidos e o grau de compressão dos mesmos. O diagnóstico preciso é essencial na escolha da modalidade terapêutica ${ }^{7}$. A sensibilidade e especificidade da imagem de ressonância magnética na investi-

1. Médico Estagiário de Cirurgia da Coluna do Hospital do Servidor Publico Estadual - São Paulo, SP, Brasil.

2. Médico Assistente do Grupo de Cirurgia da Coluna do Hospital do Servidor Publico Estadual - São Paulo, SP, Brasil.

3. Médico Chefe do Grupo de Coluna do Hospital do Servidor Público Estadual - São Paulo, SP, Brasil. 
gação do paciente com estenose de canal lombar é considerada superior aos outros exames de imagem como a mielografia e tomografia axial computadorizada ${ }^{8}$.

Os objetivos do tratamento são melhorar o grau de função e reduzir o desconforto nos membros inferiores. O tratamento conservador, através de modificações de atividades e medicações sintomáticas, demonstrou uma melhora progressiva em 15 a $43 \%$ dos pacientes durante um acompanhamento de 1 a 5 anos $^{9}$. O tratamento cirúrgico deve ser considerado em pacientes com qualidade de vida inaceitável, após esgotadas outras opções de tratamento. O objetivo principal da cirurgia é a descompressão dos elementos neurais, visando o alívio dos sintomas e o controle da progressão da estenose em pacientes com alteração neurológica progressiva e refratariedade ao tratamento clínico.

O objetivo deste estudo foi avaliar se o procedimento cirúrgico com descompressão posterior sem artrodese da coluna lombar traz benefícios aos pacientes em avaliações prospectivas de dor e incapacidade.

\section{MÉTODOS}

Foram avaliados 16 pacientes com diagnóstico estabelecido de estenose de canal lombar sintomática confirmada por meio de exame de ressonância nuclear magnética (RNM). Todos os pacientes selecionados haviam realizados tratamento com analgésicos, AINES e fisioterapia por período mínimo de seis meses sem melhora clínica satisfatória. Os critérios de inclusão foram idade acima de 65 anos, queixa principal de claudicação neurogênica, ausência de instabilidade e espondilolistese (avaliação realizada por radiografias dinâmicas). Além da RNM o exame de inclusão seria a radiografia simples, que deveria mostrar colapso discal e artrose facetária com osteofitose, sugerindo ausência de mobilidade e que a radiografia dinamica confirmasse essa observação. Os critérios de exclusão foram idade abaixo de 65 anos, neuropatia diabética diagnosticada previamente com eletroneuromiografia, insuficiência vascular associada provada por exame de Doppler arterial, doencas neurológicas crônicas como seguelas de acidente vascular cerebral, doenças neurológicas evolutivas como síndrome de Parkinson, cirurgia prévia na coluna vertebral, queixa principal de radiculopatia e presença de espondilolistese e ou sinais de instabilidade vertebral observados nas radiografias dinâmicas.

O objetivo da cirurgia foi o alívio da dor e melhora da incapacidade funcional. A cirurgia foi realizada através de um acesso posterior com laminotomia central e ressecção do ligamento amarelo, com preservação das facetas articulares para evitar instabilidade vertebral. Não foi realizada instrumentação pedicular.

Foram aplicados questionários de incapacidade e dor no período pré-operatório e durante o seguimento pós-operatório de um mês, seis meses e um ano. Para avaliação de dor, foi utilizada a escala visual analógica (EVA) com variação de 0 a 10 em intensidade. Empregou-se os questionários de incapacidade Rolland Morris e Oswestry, que relacionam incapacidade física e dor na coluna. Os questionários foram aplicados por um ortopedista especialista em cirurgia da coluna vertebral integrante do grupo de coluna do Hospital do Servidor Público Estadual de São Paulo, que também conduziu as avaliações clínicas pré e pós-operatórias.

Foi adotado o nível de significância de $5 \%(0,050)$ para a aplicação dos testes estatísticos, ou seja, quando o valor da significância calculada (p) foi menor do que 5\% (0,050), observou-se uma diferença ou relação 'estatisticamente significativa'; e quando o valor da significância calculada (p) foi igual ou maior do que $5 \%(0,050)$, identificou-se uma diferença ou relação dita 'estatisticamente não-significativa'. As análises foram produzidas nos programas SPSS (v 12.0) e no software $R$ (v.13.1).

\section{RESULTADOS}

A avaliação incluiu 16 pacientes, sendo 10 mulheres (62\%) e seis homens (38\%). A idade média foi 71,69 anos, com variação entre 65 e 82 anos.

Para descrição e comparação entre os momentos de observação, foi empregado o Teste de Friedman com o intuito de verificar possíveis diferenças entre os momentos pré-operatório, um mês, seis meses e um ano após a cirurgia, considerados variáveis lon- gitudinais em avaliações comparativas concomitantes, em relação à dor e incapacidade (Tabela 1). Foram observadas diferenças estatisticamente significativas nos medidores de dor e incapacidade.

A análise da dor através da escala EVA demonstrou valor 8,75 em média de intensidade de dor pré-operatória. Houve queda progressiva nos valores médios observados com média 2,13 após um ano de seguimento (Figura 1).

A evolução da intensidade da dor em intensa (8 a 10), moderada (3 a 7 ) ou leve ( 0 a 2 ) de acordo com a escala EVA pode ser observada na Figura 2.

A avaliação da incapacidade pelos questionários de Roland Morris (Figura 3) e Índice de Oswestry (Figura 4) mostrou queda progressiva nos valores observados durante o seguimento de um ano.

A análise da incapacidade funcional medida pelo questionário Roland Morris mostrou média de 21,13 no pré-operatório e média de 7,56 após um ano de procedimento.

A avaliação do índice de Oswestry mostrou na análise após um ano de seguimento $62 \%$ de resultados excelentes $38 \%$ de resultados bons (Figura 5).

Tabela 1. Medidas descritivas para as variáveis quantitativas.

\begin{tabular}{c|c|c|c|c|c|c|c|c}
\hline Variável & Tempo & $\mathbf{N}$ & Mínimo & Máximo & Média & $\begin{array}{c}\text { Desvio } \\
\text { Padrão }\end{array}$ & $\begin{array}{c}\text { Erro- } \\
\text { Padrão }\end{array}$ & p-valor \\
\hline Idade & & 16 & 65 & 82 & 71,69 & 4,73 & 1,182 & - \\
\hline \multirow{5}{*}{ EVA } & Pré-operatório & 16 & 7 & 10 & 8,75 & 0,93 & 0,233 & $<0,001 *$ \\
\cline { 2 - 10 } & 1 mês: pós-operatório & 16 & 2 & 5 & 3,63 & 0,96 & 0,239 & $<0,001$ \\
\cline { 2 - 10 } & 6 meses: pós-operatório & 16 & 2 & 4 & 2,56 & 0,63 & 0,157 & \\
\cline { 2 - 10 } & 1 ano: pós-operatório & 16 & 1 & 4 & 2,13 & 0,89 & 0,221 & \\
\hline \multirow{4}{*}{ Roland-Morris } & Pré-operatório & 16 & 16 & 23 & 21,13 & 1,93 & 0,482 & $<0,001$ \\
\cline { 2 - 9 } & 1 mês: pós-operatório & 16 & 10 & 14 & 12,75 & 1,29 & 0,323 & $<0,001$ \\
\cline { 2 - 9 } & 6 meses: pós-operatório & 16 & 7 & 10 & 8,50 & 0,89 & 0,224 & \\
\cline { 2 - 9 } & 1 ano: pós-operatório & 16 & 5 & 9 & 7,56 & 1,31 & 0,329 & \\
\hline \multirow{5}{*}{ Oswestry } & Pré-operatório & 16 & 0,36 & 0,78 & 0,61 & 0,10 & 0,026 & $<0,001$ \\
\cline { 2 - 9 } & 1 mês: pós-operatório & 16 & 0,20 & 0,45 & 0,37 & 0,06 & 0,016 & $<0,001$ \\
\cline { 2 - 9 } & 6 meses: pós-operatório & 16 & 0,16 & 0,36 & 0,25 & 0,06 & 0,016 & \\
\cline { 2 - 9 } & 1 ano: pós-operatório & 16 & 0,12 & 0,31 & 0,20 & 0,05 & 0,012 & \\
\hline
\end{tabular}

*Teste de Friedman para comparação de grupos relacionados (no tempo), considerando todos os tempos.

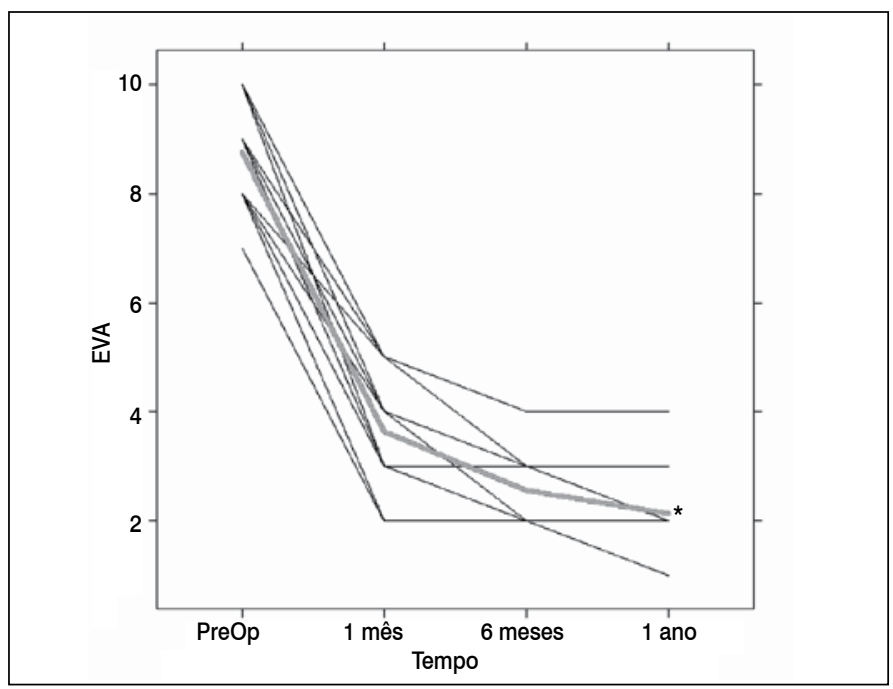

Figura 1. Perfis individuais, com a média ( ${ }^{*}$ cinza) de EVA.

\section{DISCUSSÃO}

De acordo com a literatura, a maioria dos pacientes submetidos a descompressão por estenose de canal lombar está na quinta ou sexta década de vida ${ }^{8}$. Em nossa amostra, a idade média no momento da cirurgia foi de 71,69 anos. Muitas séries demonstram um predomínio do sexo masculino de aproximadamente $56 \%{ }^{10}$. Este estudo encontrou uma relação de $62 \%$ de mulheres e $38 \%$ de homens. 


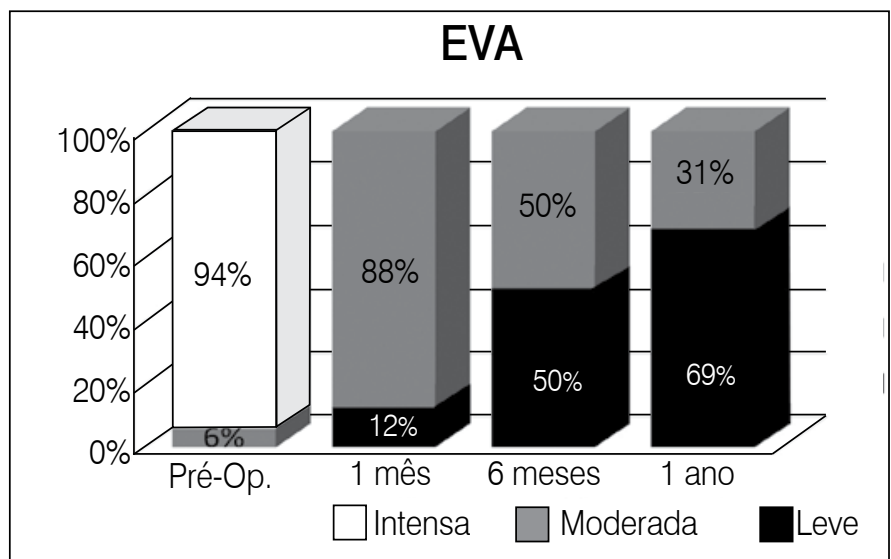

Figura 2. Evolução dos níveis da EVA ao longo do tempo.

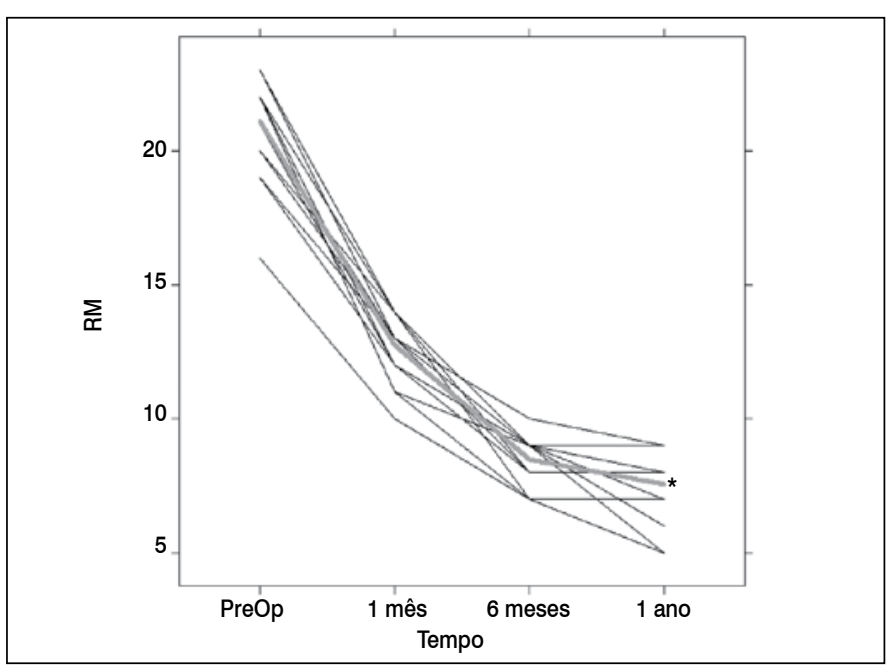

Figura 3. Perfis individuais, com a média ( ${ }^{*}$ cinza) de Roland-Morris.

$\mathrm{Na}$ análise da dor por meio da escala EVA, observou-se uma melhora significativa dos valores de pontuação de dor nas avaliações longitudinais em relação aos níveis pré-operatórios. Observou-se que todos os pacientes saíram do nível de dor intensa ainda no primeiro mês pós-operatório, mas a maioria dos pacientes ainda apresentava-se no nível de dor moderada. É possível que esse resultado esteja relacionado a morbidade inerente ao período pós-operatório recente, visto que com o seguimento houve uma queda progressiva nos níveis de dor, com a maioria dos pacientes apresentando nível de dor leve na avaliação após um ano de procedimento.

A avaliação da incapacidade funcional pelo Roland Morris e índice de Oswestry demonstrou uma melhora estatisticamente significativa nas escalas funcionais, com uma melhora mais acentuada nos primeiros seis meses de avaliação em relação ao período pré-operatório e uma melhora menos evidente nas avaliações pareadas entre seis meses e um ano pós-operatório, mas mantendo uma tendência de melhora funcional. Portanto, a descompressão

\section{REFERÊNCIAS}

1. Arbit E, Pannullo S. Lumbar stenosis: a clinical review. Clin Orthop Relat Res. 2001;(384):137-43

2. Taylor VM, Deyo RA, Cherkin DC, KreuterW. Low back pain hospitalization. Recent United States trends and regional variations. Spine (Phila Pa 1976). 1994;19(11):1207-12.

3. Fu YS, Zeng BF, Xu JG. Long-term outcomes of two different decompressive techniques for lumbar spinal stenosis. Spine (Phila Pa 1976). 2008;33(5):514-8.

4. Arnoldi CC, Brodsky AE, Cauchoix J, Crock HV, Dommisse GF, Edgar MA, et al. Lumbar spinal stenosis and nerve root entrapment syndromes. Definition and classification. Clin Orthop Relat Res. 1976;(115):4-5.

5. Kovacs FM, Urrutia G, Alarcon JD. Surgery Versus Conservative Treatment for Symptomatic Lumbar Spinal Stenosis. Spine (Phila Pa 1976). 2011;36:E1335 - E1351.

6. Weiner DK. Office management of chronic pain in the elderly. Am J Med. 2007;120(4):306-15

7. Zouboulis P, Karageorgos A, Dimakopoulos P, Tyllianakis M, Matzaroglou C, Lambiris E. Functional outcome of surgical treatment for multilevel lumbar spinal stenosis. Acta Or-

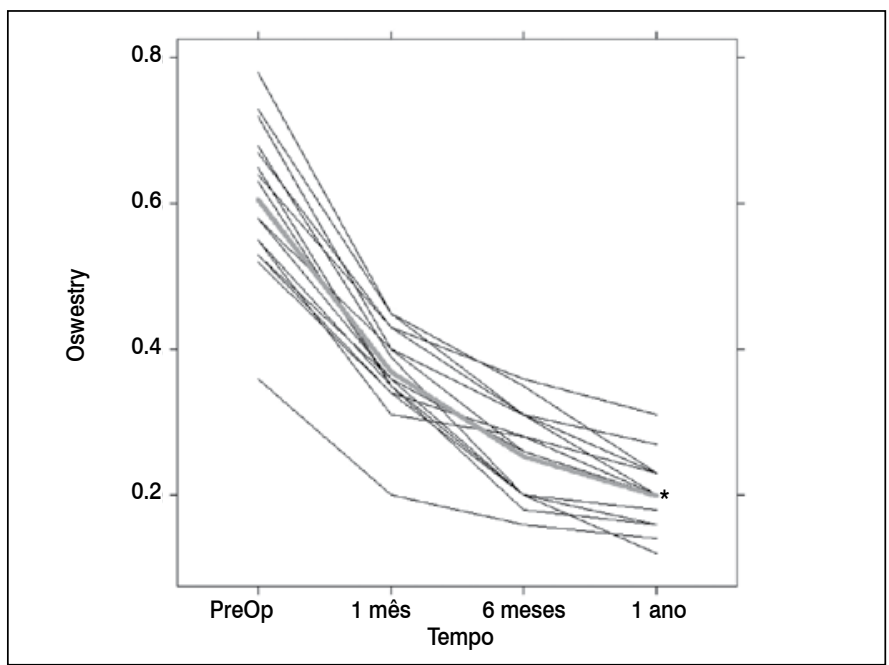

Figura 4. Perfis individuais, com a média ( ${ }^{*}$ cinza) de Oswestry.

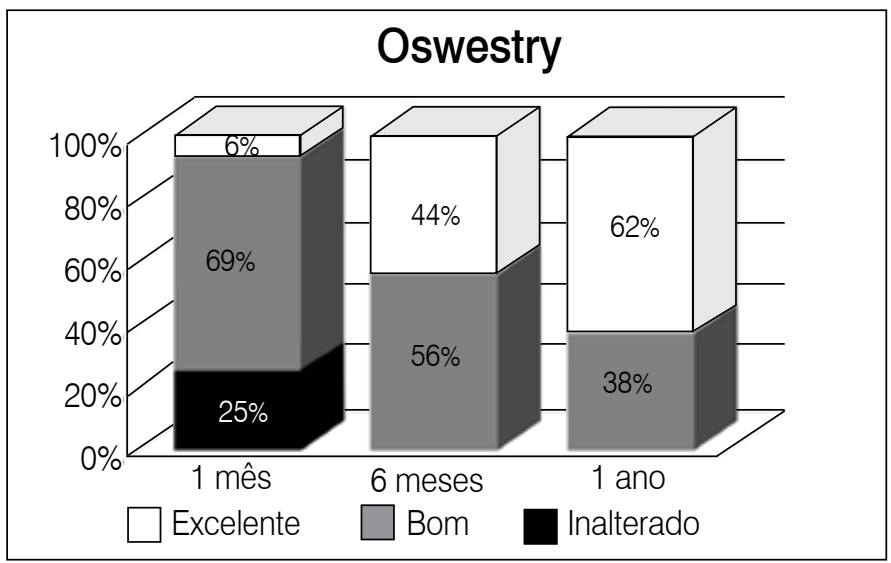

Figura 5. Índice de Oswestry ao longo do tempo.

demonstrou um impacto positivo na melhora e manutenção da qualidade de vida dos pacientes.

A história natural da ECL não é completamente conhecida devido à falta de estudos longitudinais prospectivos que documentam a evolução clínica de pacientes não-tratados ${ }^{11}$. No entanto, existem estudos que demonstram de maneira criteriosa resultados em comparações a longo prazo, evidenciando possível superioridade do tratamento cirúrgico em relação ao tratamento não-cirúrgico ${ }^{12}$. Em concordância com os resultados da literatura, acreditamos que, com indicação precisa, o tratamento cirúrgico tem fundamental importância na melhora e manutenção da qualidade de vida de pacientes com essa afecção.

\section{CONCLUSÕES}

A descompressão sem artrodese em pacientes com estenose de canal lombar demonstrou ser eficaz na melhora da dor e incapacidade em pacientes selecionados no seguimento de um ano pós-operatório.

thop. $2007 ; 78(6): 862$

8. Atlas SJ, Delitto A. Spinal stenosis: surgical versus nonsurgical treatment. Clin Orthop Relat Res. 2006;443:198-207.

9. Simotas AC. Nonoperative treatment for lumbar spinal stenosis. Clin Orthop Relat Res. 2001;(384):153-61.

10. Katz JN, Dalgas M, Stucki G, Lipson SJ. Diagnosis of lumbar spinal stenosis. Rheum Dis Clin North Am. 1994;20(2):471-83.

11. Kim JH, Lee HM, Kim HS, Moon ES, Park JO, Lee KJ, et al. Life expectancy after lumbar spine surgery: one-to eleven-year follow-up of 1015 patients. Spine (Phila Pa 1976) 2008:33(19):2116-21.

12. Atlas SJ, Deyo RA, Keller RB, Chapin AM, Patrick DL, Long MJ, et al. The Maine Lumbar Spine Study, Part III. 1-year outcomes of surgical and nonsurgical management of lumbar spinal stenosis. Spine (Phila Pa 1976). 1996;21(15):1787-94. 